\title{
SUICIDE MORTALITY IN NSW: AN INTRODUCTION TO CLINICAL AUDITS
}

Jennifer Chipps, Gavin Stewart and Geoffrey Sayer Mental Health Epidemiology

Epidemiology Branch

Public Health Division, NSW Health Department

T his is the second of a series of articles on the epidemiology of suicide in NSW by the Mental Health Epidemiology Group (MHEG) 1 . In the first article, clinical audit was suggested as a possible way of meeting the need to address suicide issues at a local level ${ }^{2}$. This article is an introduction to the use of clinical audit to identify possible preventive approaches to suicide. We examine the ethical issues, techniques and feasibility of this method for collecting information on suicides. A more detailed report on the practicalities of clinical audit is being prepared ${ }^{3}$.

For more than three decades psychological autopsies and modified psychological autopsies have been employed worldwide to study risk factors for suicide. The term psychological autopsy most commonly refers to interviews with family and friends of the victim to reconstruct the circumstances of the suicide ${ }^{4}$. Sometimes the term is limited to the determination of the mental state of the individual, and at other times the modified psychological autopsy or clinical audit includes all the investigations relevant to the suicide, including the review of medical records and the physical autopsy4.

Clinical audit in this article encompasses both the psychological autopsy and the physical autopsy, and any other relevant investigations. We prefer to use the term clinical audit to stress that interviewing bereaved people requires clinical skills and because attention should be given to the potential role clinical services may play in prevention.

The purpose of a clinical audit is not only to "explore and identify contributing factors to suicide and attempts", but also to have a formal method for "... initiating contact and effective follow-up with bereaved family and friends" and to "prevent future suicides in bereaved families and friends by being available as a point of contact for support education and counselling",5.6. It is thus a form of psychosocial research which inevitably includes a clinical component.

\section{ETHICAL CONSIDERATIONS}

Beneficence and non-maleficence: In utilising clinical audit as a therapeutic and a data collection tool, the benefit for the informants, the psychological strain on the interviewer ${ }^{\top}$ and the benefit to the community should all be considered. Suicide is a traumatic event and bereaved survivors may still show signs of crisis reactions. A clinical audit may be a further traumatic experience. However, a review of psychological autopsies by Beskow ${ }^{4}$ reported that when survivors were approached to be interviewed there were few who refused and there were no reports of the people interviewed being harmed by the interview. Many of those interviewed reported benefiting from $\mathrm{it}^{4,8}$. In a study on youth suicide 4 , researchers identified that 59 per cent of survivors had unsatisfactory bereavement adjustment outcomes and 31 per cent displayed signs of depression. Interviews designed with due respect for the needs of survivors provide the opportunity to deal with those issues. Well-planned clinical audit can be an integral part of the bereavement counselling process.

Autonomy: It is essential that the autonomy of the informants be respected. Appropriate procedures for clinical audit must be developed and staff conducting clinical audits must be properly trained. From the first approach to the last contact, informants must be confident that their participation is valued, their willingness or refusal to participate is respected, their disclosure or non-disclosure of sensitive information is entirely free ${ }^{4}$, that confidentiality of information is formally guaranteed, and that they understand the purpose of the audit.

Public good: Only the collection of the best possible information in the most rigorous manner can justify the intrusion of a clinical audit. Data quality is thus a primary ethical issue.

\section{METHOD}

What information can be collected? Schneidman ${ }^{9}$ details data to be collected according to three questions: "Why did the individual do it? How did the individual die? What was the most accurate mode of death - accident or suicide?" Clearly, information also needs to be collected which may facilitate the role of clinical services in prevention. A useful outline is suggested by Spellman and Heyne ${ }^{10}$ :

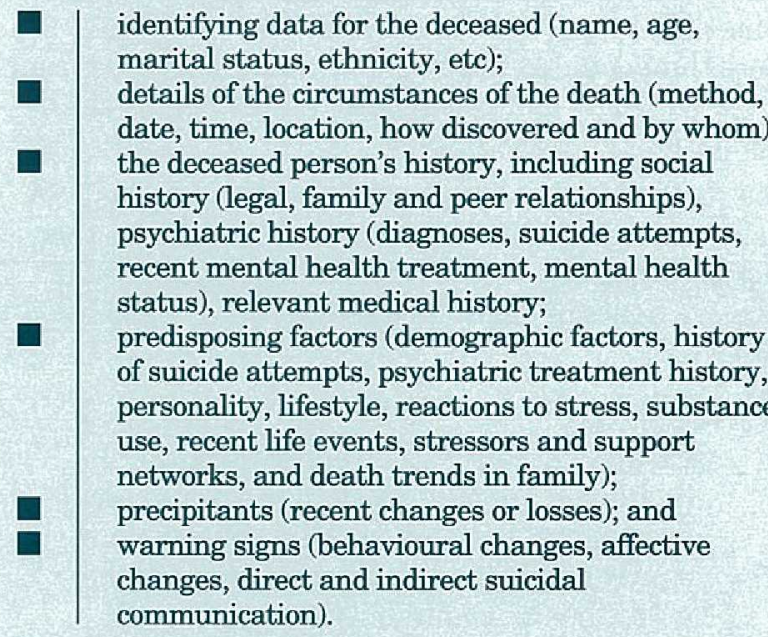

It is important to integrate this data with information from biochemical, toxicological and epidemiological approaches ${ }^{11,12}$.

Table 6 compares the information obtained in a clinical audit versus other collections.

Standard data collections, such as the ABS death data and the ISC hospital separation data, provide only limited information on the risk factors contributing to suicide. In contrast, clinical audits explore in detail the immediate history of the suicide, including information not routinely collected. Although not essential, case control studies can also be conducted as an adjunct to the clinical audit to quantify the effects of the risk factors ${ }^{26,31}$.

\section{From whom should information be collected?} Information should be sought from knowledgeable informants. These include the relevant health workers as well as first or second degree relatives or close friends. In three studies where alternative informants were available, the proportion of cases with acceptable interview data ranged from 89 per cent to 100 per cent $t^{4}$. The refusal rate recorded in two of the studies was only 7 per cent and 9 per cent. In an audit of suicide in the elderly, a review of the 


\section{TABLE 6}

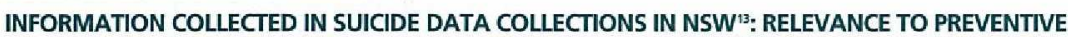
APPROACHES AND THE EXTENT TO WHICH THESE ARE AVAILABLE IN VARIOUS DATA SOURCES

\begin{tabular}{|c|c|c|c|c|}
\hline $\begin{array}{l}\text { Data Collection } \\
\text { Identifying Information: }\end{array}$ & $\begin{array}{l}\text { Australian Bureau } \\
\text { of Statistics (ABS) } \\
\text { Death Data }^{14}\end{array}$ & $\begin{array}{l}\text { NSW Mental } \\
\text { Health Client } \\
\text { Suicide Incident } \\
\text { Monitoring System }{ }^{15}\end{array}$ & $\begin{array}{l}\text { Inpatient Statistics } \\
\text { Collection (ISC) } \\
\text { separation data } \\
\text { (attempts) }\end{array}$ & Clinical audit \\
\hline Intent ${ }^{16}$ & $\checkmark$ & $\checkmark$ & $\checkmark$ & $\checkmark$ \\
\hline Demographics & $\checkmark$ & $\checkmark$ & $\checkmark$ & $\checkmark$ \\
\hline Psychiatric diagnoses & & $\checkmark$ & $\checkmark$ & $V$ \\
\hline Psychiatric history $5,17,18,19,20,21,22,23,24$ & & & & $\checkmark$ \\
\hline Personality ${ }^{5,25,26}$ & & . & & $\checkmark$ \\
\hline Method, location and time & $\checkmark$ & $\checkmark$ & $\checkmark$ & $\checkmark$ \\
\hline Risk factors ${ }^{18.25}$ & & & & $\checkmark$ \\
\hline Life events ${ }^{21}$ & & & & $\checkmark$ \\
\hline Social support ${ }^{25,27}$ & & & & $\checkmark$ \\
\hline Interpersonal stressors ${ }^{27,28}$ & & & & $\checkmark$ \\
\hline Precipitants & & & & $\checkmark$ \\
\hline Family history ${ }^{5}$ & & & & $\checkmark$ \\
\hline Physical comorbidity ${ }^{19,21,29,30}$ & $\checkmark$ & & $\checkmark$ & $\checkmark$ \\
\hline Health care contacts $s^{21,22,29,31}$ & & $\checkmark$ & & $\checkmark$ \\
\hline Previous attempt & & $\checkmark$ & & $\checkmark$ \\
\hline Warning ${ }^{5,21}$ & & $\checkmark$ & & $\checkmark$ \\
\hline Preventability & & & & $\checkmark$ \\
\hline
\end{tabular}

availability of knowledgeable informants found there was at least one knowledgeable informant in 90 per cent of the cases and two or more in 50 per cent of cases $^{32}$.

The main technical disadvantage of the clinical audit lies in the retrospective nature of data collected. Both the interviewer and the informants are aware the person has committed suicide and recall may be distorted by this knowledge ${ }^{18}$. It is therefore important to conduct the interview and record the interview in a structured manner.

Who should collect the information? It is sometimes suggested the audit be conducted by the health worker/s responsible for the care of the person who committed suicide $^{6}$. But few people who commit suicide are under the immediate care of a health worker. More generally it is suggested interviews be conducted by researchers who should have relevant clinical experience to enable them to deal with people in grief and assist the individual in gaining access to appropriate services ${ }^{10}$. An alternative model is a clinical audit or review committee consisting of three or four impartial investigators who are qualified multidisciplinary mental health practitioners ${ }^{10}$.

When should the interview be conducted? There should be a fine balance in timeliness when considering the grieving process and willingness to discuss the event, and the quality of the data. A short time may increase the therapeutic potential of the interview as well as the risk of harm. On ethical grounds, it has been suggested interviews should not take place until four weeks after the suicide $e^{4}$.

To ensure reliability of the data collected, most of the studies in the literature reviewed recommend no more than a six-month interval before the interview ${ }^{4,733}$. Most studies reviewed allowed a period of eight weeks and in one study where the median time interval was nine weeks, 41 per cent of those interviewed said they would have preferred an earlier interview'. Some provision should clearly be made for people who wish to have earlier contact with the clinical audit team.

How is the information collected? Contact by telephone followed by an introductory letter is recommended. This approach has a low rejection rate and provides an opportunity to meet survivors in an empathic manner for first contact ${ }^{\top}$. Techniques for data collection include: questionnaires using standardised schedules, taping of interviews ${ }^{7}$, review of health records and review of coroners' records. It is important to collect this information in a formal and structured way.

How valid and reliable is the information collected? Despite differing methods of approach to families and interview techniques, studies show high compliance and consistency of results across a wide age range and geographic samples ${ }^{33,34}$. Although the psychiatric diagnoses assigned show a high level of validity ${ }^{13,34}$ and reliability, this approach to assigning psychiatric diagnoses is likely to have a higher specificity than sensitivity ${ }^{34}$.

No literature was found on the validity and reliability of other information collected by this method. 


\section{Suicide mortality in NSW}

Continued from page 69

Would it be feasible to conduct clinical audits on all suicides in NSW? Suicide is a relatively rare event. In NSW in 1992 there were 740 deaths by suicide ${ }^{35}$. The average burden for an Area Health Service with the highest number of suicides lies within the range of 3-13 a month an average of seven clinical audits a month ${ }^{36}$. Some of the smaller District Health Services have one suicide a year and it would be beneficial for these Districts to combine their efforts in conducting clinical audits.

Some mental health services already conduct reviews of suicides and serious suicide attempts by mental health clients as part of the post-suicide management protocol. These reviews are conducted to debrief staff and relatives formally and to evaluate health service issues for improved management of mental health clients. They do not usually include systematic data collection on suicide risk factors from the bereaved relatives and, if counselling of bereaved relatives is required, information is collected on an ad hoc basis.

The clinical audit, including interviews with the bereaved relatives, could therefore be structured as part of the postsuicide management protocol. This could be done not only for mental health clients, but also extended to all suicides.

If the clinical audit process were extended to include all serious suicide attempts (an estimated 3,536 attempts resulting in hospitalisation in NSW in $1992^{37}$ ), it would have much greater resource and workload implications.

\section{CONCLUSION AND RECOMMENDATIONS}

Clearly there are well established procedures for conducting clinical audits for suicide and many important design factors of such a system are well defined. Setting up clinical audits for all suicide deaths in NSW and exploring the immediate preceding histories to identify possible preventive approaches is thus feasible in principle. The burden for each Area and District is probably manageable and, if the clinical audit is part of a protocol for the postsuicide management, it would be a valuable source of information for the Mental Health Services. It would enable them to identify appropriate intervention and prevention strategies, provide staff education and support, and provide counselling to the bereaved. Such an approach could be developed at a local level, address local problems and visibly meet community expectations that suicide deaths are given serious consideration.

1. Membership of MHEG is open to people with professional interest and expertise in mental health epidemiology who are willing to contribute to the planning and production of a series of publications and reports on important mental health topies. The policy of MHEG is joint publication by the group as a whole in which authors are listed in order of their contribution to the particular report. The contact address for MHEG is Mental Health Epidemiology Group, Centre for Clinical Policy \& Practice, Public Health Division, NSW Health Department, Locked Bag 961, PO North Sydney 2059. (Fax: 391 9041, Internet email jchip@gwsm.doh.health.nsw.gov.au).

2. Stewart G, Chipps J, Sayer G. Suicide mortality in NSW: geographic variations. NSW Public Health Bulletin 1995; 6(6):49-51. 3. A more detailed report which will review the practicality of implementation of clinical audits on a State level is being prepared. Protocols for data to be collected, ethical and legal issues affecting the audit and the feasibility of implementing this on a local level will be discussed. Suggestions are invited.

4. Beskow J, Runeson B, Asgard U. Ethical aspects of psychological autopsy. Acta Psychiatry Scandinavica 1991; 84:482-487. 5. Shafii M, Carrigan S, Whittinghill JR, Derrick A. Psychological autopsy in children and adolescents. Am J Psychiatry 1985; 149(9):10611064.

6 . Kaye NS, Soreff SM. The psychiatrist's responses and responsibilities when a patient commits suicide. Am J Psychiatry 1991; 148:739-743. 7. Beskow J, Runeson B, Asgard U. Psychological autopsies: methods and ethics. Suicide and Life Threatening Behaviour 1990; 20(4):307-323.

8. Westrin G, Nistun T, Smedly B, Haglund B. Epidemiology and moral philosophy. J Med Ethics 1992; 18:193-196.

9. Little JD. Staff responses to inpatient and outpatient suicide: what happened and what do we do? Aust NZ J Psychiatry 1992; 26:162-167. 10. Spellman A, Heyne B. Suicide? Accident? Predictable? Avoidable? The psychological autopsy in jail suicides. Psychiatric Quarterly 1989 60(2): 173-183.

11. Beskow J, Thoron J, Ostrom M. National suicide prevention programme and railway suicide. Social Science and Medicine 1994; 38(3):447-451.

12. Busuttil A, Obafunwa JO, Ahmed A. Suicidal inhalation of vehicular exhaust in the Lothian and Borders region of Scotland. Human Experimental Toxicology 1994; 13(8):545-550.

13. Note - The Australian Coroners Society has endorsed the recommendations of the report: Coronial Information Systems: needs and feasibility study, J Moller, National Injury Surveillance Unit, Adelaide, 1994 to develop a coroners' database. This is not operational yet.

14. Note - There is a two-year delay in receiving death data from the ABS. 15. Note - The NSW Suicide Notification and Incident Monitoring

System has been operating for 41 months. The system has collected data on suicide of clients of the public mental health services. The system is being reviewed and a report on the existing data as well as the review will be available soon.

16. Cantor CH, Brodie J, McMillan J. Firearm victims - who are they? Med J Aust 1991; 155:442-445.

17. Rao U, Weissman MM, Martin JA, Hammond RW. Childhood depression and risk of suicide: A preliminary report of a longitudinal study. $J$ Am Academy of Child and Adolescent Psychiatry 1993; 32(1):21-27. 18. Brent DA, Perper JA, Moritz G et al. Psychiatric risk factors for adolescent suicide: a case control study. J Am Academy of Child and Adolescent Psychiatry 1993; 32(3):521-529.

19. Berglund M, Krantz P, Lundqvist G. Suicide in alcoholism: a prospective study in 55 cases with autopsy findings. Acta Psychiatrica Scandinavica 1987; 76:381-385.

20. Lesage $A D$, Boyer R, Grunberg $F$ et al. Suicide and mental disorders: a case control study of young men. Am J Psychiatry 1994; 151(7):1063-1068. 21. Asgard U. A psychiatric study of suicide among urban Swedish women. Acta Psychiatric a Scandinavica 1990; 82:115-124. 22. Isometsa ET, Henriksson MM, Aro HM et al. Suicide in major depression. Am J Psychiatry 1994; 151(4):530-536.

23. Henriksson MM, Aro HM, Martunnen MJ et al. Mental disorders and comorbidity in suicide. Am. J Psychiatry 1993; 150(6):935-939.

24. Martunnen MJ, Aro HM, Henriksson MM, Lonnqvist JK. Mental disorders in adolescent suicide. DSM-III_R Axes I and II diagnoses in suicides among 13-to 19-year-olds in Finland. Arch Gen Psychiatry 1991; 48:834-839.

25. Martunnen MJ, Aro HM, Henriksson MM, Lonnqvist JK, Antisocial behaviour in adolescent suicide. Acta Psychiatrica Scandinavica 1994; 89:167-173.

26. Duberstein R, Conwell Y, Caine ED. Age differences in the personality characteristics of suicide completers: preliminary findings from a psychological autopsy study. Psychiatry 1994; 57:213-223. 27. Martunnen MJ, Aro HM, Henriksson MM, Lonnqvist JK. Precipitant stressors in adolescent suicide. J Am Academy of Child and Adolescent Psychiatry 1993; 32(6):1178-1183.

28. Duberstein R, Conwell Y, Caine ED. Interpersonal stressors, substance abuse, and suicide. I Nervous and Mental Disease 1980; 181(2):80-85

181(2):80-85. suicide in elderly men. Hospital and Community Psychiatry 1992; 43(12):1198-1203.

30. Conwell Y, Caine ED, Olsen K. Suicide and cancer in late life. Hospital and Community Psychiatry 1990; 41(12):1334-1338.

31. Isometsa ET, Henriksson MM, Aro HM, Lonnqvist JK. Suicide in bipolar disorder in Finland. Am J Psychiatry 1994; 151(7):530-536. 32. Younger SC, Clark DC, Oehmig Lindroth R, Stein RJ. Availability of knowledgeable informants for a psychological autopsy study of suicides committed by elderly people. J Am Geriatric Society 1990; 38(11):11691175.

33. Little JD. Staff responses to inpatient and outpatient suicide: what happened and what do we do? Aust NZ J Psychiatry 1992; 26:162-167. 34 . Brent DA. The psychological autopsy: methodological considerations for the study of adolescent suicide. Suicide Life Threatening Behaviour 1989; Spring 19(1):43-57.

35. ABS Death Data, 1992

36. Northern Sydney Area Health Service - 85 cases registered - ABS Death Data, 1992

37. NSW Inpatient Statistics Collection separations, 1992. 